\title{
Spatially resolved isotope labeling
}

\section{Electron-energy-loss spectroscopy with a scanning transmission electron microscope enables spatially resolved imaging of isotope-labeled molecules.}

\author{
$\mathrm{n}$ biological experiments, isotope labeling \\ enables scientists to track molecules \\ through biochemical pathways. For this, \\ specific atoms in a molecule are replaced \\ by alternative isotopes-for example, \\ ${ }^{13} \mathrm{C}$ might replace ${ }^{12} \mathrm{C}$. Isotope labels are \\ detected via mass spectrometry, which \\ involves the destruction of the sample and \\ the loss of spatial information. In contrast, \\ tip-enhanced Raman spectroscopy (TERS) \\ and scanning near-field optical microscopy \\ (SNOM) allow the detection of vibrational \\ spectra of biomolecules with high spatial \\ and spectral resolution while preserving \\ the sample. \\ As a complementary method to \\ TERS and SNOM, electron-energy-loss \\ spectroscopy (EELS) also enables high \\ spatial resolution of vibrational modes in \\ solid materials. However, the high electron
}

doses needed are not compatible with imaging of biological materials. Vibrational EELS also works in an 'aloof' mode in which the electron beam is positioned a few nanometers away from the sample to avoid sample degradation.

Juan Carlos Idrobo and colleagues from the Oak Ridge National Laboratory have now developed a method that combines 'aloof' monochromated EELS with an aberration-corrected scanning transmission electron microscope to analyze the isotope-labeled amino acid L-alanine. To demonstrate their method, the authors first imaged ${ }^{12} \mathrm{C}$ - and ${ }^{13} \mathrm{C}$-labeled alanine crystals separately. They then imaged crystals of both alanines in the same sample, and confirmed the capability of their method to distinguish between the two compounds with nanoscale precision via Fourier transform infrared spectroscopy. The different L-alanine clusters were separated by several hundreds of nanometers, but the researchers estimate that it is possible to resolve structures just $50 \mathrm{~nm}$ apart from each other.

In combination with cryogenic sample preparation, the method could also be used to track how isotope-labeled molecules are processed in metabolic pathways in whole-cell samples, for studies of these processes in their cellular environment.

\section{Christian Schnell}

Published online: 28 March 2019

https://doi.org/10.1038/s41592-019-0379-x

\section{Research papers}

Hachtel, J. A. et al. Identification of site-specific isotopic labels by vibrational spectroscopy in the electron microscope. Science 363, 525-528 (2019).

\section{We Create Solutions}

Ultra Precise Motion Control - D.C. Servo motors down to $20 \mathrm{~nm}$, piezos down to $1 \mathrm{~nm}$, and low drift XYZ stages.

Microscopy - Automation, modular microscopes, autofocus complete light sheet systems, and components.

OEM - Custom designed systems to user specifications.

www.asiimaging.com

info@asiimaging.com

(800) $706-2284$ or (541) 461-8181

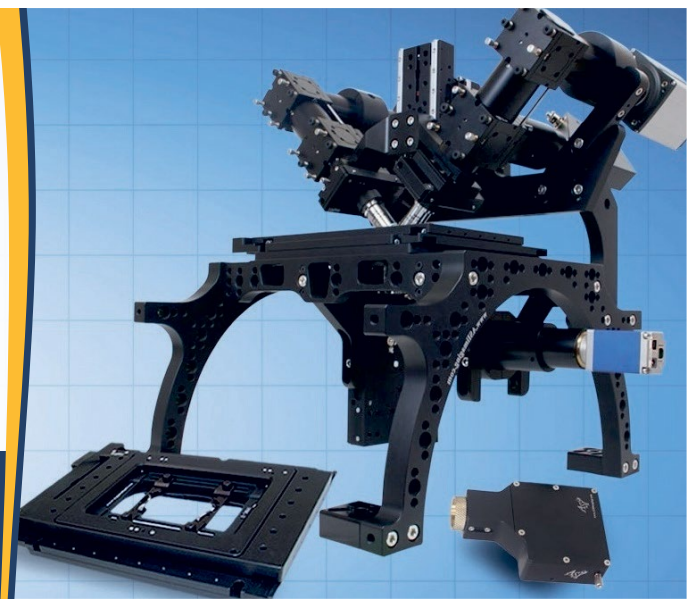

